\title{
On the Formation, Replication and Transmission of Strong Memes and their Cognitive Psychological Motivations*
}

\author{
Yongmei Jiang \\ Qingdao University of Science and Technology, Qingdao, China \\ Email: yongmeijiang716@126.com
}

\begin{abstract}
This article first advances a fact that a Chinese word geili has suddenly become very popular and known to almost every one. It then tries to explain the reasons that it has become so hot in such a short time. The author tries to explain this phenomenon with the meme theory, and summarizes the characteristics of strong memes. Then it dwells on the cognitive psychological motivations that influence the formation, replication and transmission of strong memes.
\end{abstract}

Index Terms - strong memes, characteristics, cognitive psychological motivations

The Chinese word Geili (给力, pronounced as "gěi lì") is from a northern Chinese dialect, and was scarcely known by most Chinese people several years ago. But in the last few years, it suddenly caught people's attention and has become populous among the Netizens at first, and then became ubiquitous, including newspapers, advertisements and TV and radio programs and almost every thing that needs to attract people's attention, even appeared in many serious newspapers, for example China Daily, one of the most serious newspapers in China. And now it has come to people's everyday speech.

On April 27, 2011, the author put it in the searching engine Baidu and instantly got about one million relevant outcomes, from which we can easily see how popular it has become. From these outcomes, the author carefully picked 100 sentences, then analyzed and classified them.

The result shows that it mainly have two parts of speech.

First of all, as adjective, meaning "good, supportive, strong, powerful, cool, awesome, etc", for example, on class the students may be saying “这课上得好枯燥, 不给力呀! ”meaning “This lecture is not interesting.” and in the company when the boss is more generous about the employees' salary, the clerks might talk with each other like "这BOSS真给力 啊! ” meaning "this boss is very good and generous".

People soon coined an English word for it, which is "geilivable", and it's opposite word is "ungeilivable", meaning "something is far from what you have expected, disappointing, not good".

Second, as verb, which means “to bring strength, or cheer up”, for instance, “要给力啊, 后期靠你了” meaning to call on some one to work hard because he is depended on by every one.

Exactly how do ideas spread, and what are the factors that make them genuine thought contagions? Why, for instance, do some beliefs spread throughout society, while others dwindle to extinction? How could this word suddenly be accepted by so many people and become so hot? If we could find out the reason, may be we could use it to make other things just as acceptable and that might be a great contribution to many fields, for example, the advertisement or language learning. The author has done a lot of research and referred to many books and magazines in order to find an explanation for this. At last, she found the meme theory, which can very properly explain this phenomenon.

The meme theory is a new theory to explain the formation and development of language. Its replication and transmission provide a quick and an effective way to enrich advertising slogans. The paper tries to explain the characteristics and cognitive psychological motivations for strong memes.

\section{Memes And Memetics}

In 1976, Richard Dawkins, a British zoologist from the Cambridge University advanced the famous Meme Theory in his book The Selfish Gene, and applied it into the practice of explaining culture. Since then, many western scholars began to do innovative research by combining the meme theory with a lot of subjects, including old as well as new ones, broking them down and then compounding them in different new ways. They have developed a fairly large scale of cross-disciplinary research with the meme theory as the main thinking train. In recent years, many Chinese scholars began to introduce, interpret, study and demonstrate this theory and combine it with many subjects in culture, language, language teaching and various other fields. They have done a lot of research and achieved remarkable success.

Heylighen (1992) argues that meme has to experience four different stages from the moment it is selected by a new

\footnotetext{
${ }^{*}$ This research is funded by Qingdao University of Science and Technology, and the project number is 10XC21.
} 
master and is afterward passed on, which consists of assimilation, retention, expression and transmission. The most attractive, most vital, most infective ones with the fastest spreading speed and hugest influence were named strong memes by him.

The Meme Machine (Blackmore, 1999) is a popular science book by psychologist Susan Blackmore on the subject of memes. Blackmore attempts to constitute memetics as a science by discussing its empirical and analytic potential, as well as some important problems with memetics. The first half of the book tries to create greater clarity about the definition of the meme as she sees it. The last half of the book consists of a number of possible memetic explanations for such different problems as the origin of language, the origin of the human brain, sexual phenomena, the internet and the notion of the self. These explanations, in her view, give simpler and clearer explanations than trying to create genetic explanations in these fields.

Effective memes will be those that cause high fidelity, long-lasting memory. Memes may be successful at spreading largely because they are memorable rather than because they are important or useful. An important task of memetics will be to integrate the psychology of memory with an understanding of memetic selection.

Chinese scholar He Ziran (2005) believes that memes actually refer to cultural memes. It exists by transmission and language is one of it's carriers. Meme is propitious to the development of languages and meme itself depends on the replication and transmission of languages. From the point of view of memetics, language memes discover the law of the spread of speeches and the transmission of languages. Memes in natural languages are expressed in three aspects: education and the teaching of knowledge, the exploitation of language itself and the communication and intercourses through information. He Ziran advocated (2008) that as a meme, language's vitality depends on weather it could be accepted during the process of being used and in turn be widely copied and transmitted. Usually the most widely copied and transmitted memes are strong memes.

\section{CHARACTERISTICS OF STRONG MEMES}

Though each brain's structured capacities are different, there must be some commonality in the basic cognitive apparatus of human beings which allow some memes to be more easily assimilated and retained. Thus, it is a crucial task to seek the common features and constraints of human mind and make memes more adapted to them.

In this section, we explore several principles which are more memorable and cognitive and make strong memes, but they are not for completeness or exhaustiveness, just for suggestion and illumination.

Cognitive psychologist George A, Miller (1956), argued that human being's working memory is severely limited in size, for it can only hold approximately 5-9 chunks of information (seven plus or minus two). Chunk is any meaningful unit, which can be a digit, a word, a saying, a poem, and so on. Its capacity depends on people's knowledge and experience. To cope with the constraints of working memory, writers and speakers are suggested to follow two principles: first, strong memes are often short and terse; second, the capacity of a strong meme is often enlarged by chunking, which is a way of grouping individual pieces of information into larger units.

Obviously the word geili has become a strong meme, and it is not accidental but inevitable, because it has almost all the striking characteristics of a strong meme. For example:

1 Novelty. Speeches with original and special structures could better capture people's attention. In this highly developed internet and information age, there is a universal tendency of seeking novelty, and fancy words could attract people's eyes or ears instantly.

2 Sensibility thrill. Compared with common language, memes with sensibility thrill have stronger infection and vitality, and will be bitterly received after having got into the accepter's brain.

3 Terse. It can also be called short or economic. Such memes could spread with very fast speed and could become popular in a large scope within a short time, because they save time and energy at least. Furthermore, their popularity will increase with the pass of time.

4 Expressiveness. A strong meme must be very expressive in order to be accepted by both the users and the readers and listeners. Only when it has this character, the users would want to resort to it and the others would readily accept it.

5 Easy to accept. They already have certain information foundation among the receivers, i.e. people are familiar with their original structures which the disseminator has only varied and copied directly but applied them in totally different context. This creative application of memes acquired very good effect. They spread very fast because they are easy to understand and remember.

6 Easy to imitate. Memes spread mainly through imitation and only the ones that could easily be imitated are able to transmit quickly, and therefore become strong memes.

7 Having a stable structure. To exist in people's communication for a long period, a meme must have a relatively stable structure, and the transmission of memes is based on certain structures. As we all know, during the transmission, memes could show variations. But no matter how memes vary, the varied meme and the original meme structure have at least one thing in common in order to sustain the certain relationship between them. The community they formed could be called meme field and all the memes in the same field have one common characteristic, which is similar meme structure. That is to say, the same and similar structure is the most basic requirement to sustain the meme field, and it is the foundation for the transmitters and accepters to resonant with each other during the process of transmission. The essence of people replicating memes is virtually the replication of it's structure. 
8 Of informal Style. To create strong headline memes which are easily understood by readers and listeners, writers and speakers must first of all make negotiable choices on code and style. According to Verschueren (He Ziran, 1999), style can be reserved to describe variability along dimensions of formality and informality, range from casual or colloquial speech to highly formal uses.

Looking back on the successful and classical meme home and abroad, most of them are novel, short, direct, colloquial, easy to accept and closer to everyday speech. Here we regard this informal style as "a strong meme of form" in the field of headline delivered in this form can not only be easily understood, but also be effortlessly expressed, and ultimately become more popular.

The eight characteristics concluded above are exterior presentations shown by strong memes. To study the reasons that some memes could become strong ones, we need to penetrate into the interior and focus on its cognitive reasons.

\section{Cognitive Psychological Motivations}

When language memes are studied, their cognitive psychological motivations shouldn't be neglected. Cognitive psychology is one of the main reasons that people take specific actions, including physical activities and speeches. Memes become one of the main means that people spread and perceive the world, which is also brought about by people's specific cognitive psychology. Some cognitive methods may be able to explain this phenomenon. For example:

1 flashbulb memory. It is highly detailed, exceptionally vivid "snapshots" of the moment and circumstances in which surprising and consequential (or emotionally arousing) news was heard. It has six characteristic features: place, ongoing activity, informant, own affect, other affect, and aftermath. It is stored on one occasion and retained for a lifetime. Usually, such memories are associated with important historical or autobiographical events. For example, many people in the US who were adults in the 1960s have flashbulb memories for the assassinations of President Kennedy and Martin Luther King, and can recall in elaborate detail when and how they heard the news. (Younger Americans sometimes have flashbulb memories for the explosion of the spaceship Challenger.) By contrast, few people have detailed memories of events which happened the day before or after each assassination. People also may form flashbulb memories of important personal events, such as hearing about the death of a family member or witnessing an unusual trauma such as a disaster. It is believed to be an appropriate name for the phenomenon in that it suggests surprise, an indiscriminate illumination, and brevity. The name is inappropriate, however, in that an actual photograph, taken by flashbulb, is indiscriminate and preserves everything within its scope. Flashbulb memories, in actuality, are only somewhat indiscriminate and are far from complete.

What makes the flashbulb memory special is the emotional arousal at the moment that the event was registered to the memory. It increases the ability to recall the details of the event and tend to be retold over and over again.

2 Short-term memory (or "primary" or "active memory") is the capacity for holding a small amount of information in mind in an active, readily available state for a short period of time. The duration of short-term memory (when rehearsal or active maintenance is prevented) is believed to be in the order of seconds. A commonly cited capacity is $7 \pm 2$ elements.

Whatever the cause or causes of forgetting over the short term may be, there is consensus that it severely limits the amount of new information that we can retain over brief periods of time. This limit is referred to as the finite capacity of short-term memory. The capacity of short-term memory is often called memory span, in reference to a common procedure of measuring it.

Capacity of STM can be affected by the following: Influence of long-term memory, Reading aloud, Pronunciation time and so on.

3 human beings have an innovative tendency. It can also be called the psychology of seeking discrepancy. It means people want to create and break rules and they have a psychological tendency to deal with and solve problems in a unique way. The transmission of most memes is caused by this specific innovative tendency of human beings. A lot of researches show that every one has creativity, but some people's are recessive and not shown. But in their subconscious, they are willing to accept original things.

The time-worn question of conservatism as against change has evidently much in common with that of personality as against institutions. Innovation, that is, is bound up with the assertion of fresh personality against mechanism; and the arguments for and against it are the same as I have already suggested. Wherever there is vigor and constructive power in the individual there is likely to be discontent with the establishment. The young notoriously tend to innovation, and so do those of a bold and restless temperament at any age; the old, on the contrary, the quiet, the timid, are conservative. And so with whole peoples; in so far as they are enfeebled by climate or other causes they become inert and incapable of constructive change. Innovation or the opposite may be a public habit, independently of differences in age or vigor. The attitude toward change is subject to the same sort of alteration as public opinion, or any other phase of the public mind(Cooley, 1909).

People could feel aesthetic fatigue toward prosaic and dull things and are not ready to pay more attention to them. On the contrary, creative things are easy to be accepted by most people. The transmission of memes is controlled by this psychology. In speech intercommunication, common memes can't satisfy people's creative and discrepancy seeking tendency, therefore can't get people's attention. As a result, people change common memes and create original, unique memes which could better satisfy the need of reality.

Memes evolve by natural selection in a process similar to that of Genes in evolutionary biology. What makes an idea a 
potent meme is how effectively it out-propagates other ideas. In memetic evolution, the "fittest ideas" are not always the truest or the most helpful, but the ones best at self replication. Thus, crash diets spread not because of lasting benefit, but by alternating episodes of dramatic weight loss and slow regain. Each sudden thinning provokes onlookers to ask, "How did you do it?" thereby manipulating them to experiment with the diet and in turn, spread it again. The faster the pounds return, the more often these people enter that disseminating phase, all of which favors outbreaks of the most pathogenic diets. Like a software virus traveling on the Internet or a flu strain passing through a city, thought contagions proliferate by programming for their own propagation. Lynch (1996) argues that certain beliefs spread like viruses and evolve like microbes, as mutant strains vie for more adherents and more hosts. In its most revolutionary aspect, memetics asks not how people accumulate ideas, but how ideas accumulate people.

Therefore, it is not accidental for strong memes like geili to be widely copied and transmitted, but has certain characteristics necessary for it to be widely accepted and is a social phenomenon according with the universal cognitive psychological foundation of human beings. Therefore, it can be used by advertisement makers or teachers to make a slogan widely accepted or to impress the audience.

\section{REFERENCES}

[1] Heylighen, F. (1992). Selfish Memes and the Evolution of Cooperation, Journal of Ideas, 24, 77-84.

[2] Blackmore, S. (1999). The Meme Machine, Oxford: Oxford University Press.

[3] Dawkins, R. (1976). The Selfish Gene, New York: Oxford University Press.

[4] He Ziran, (2005). Memes in Languages, Language Science, 6, 200-209.

[5] He Ziran, (2008). Language Memes and Their Rhetoric Effect, Foreign Language Research, 1, 68-76.

[6] He Ziran, (1999). Understanding Pragmatics-A Review Article of Linguistic Choice, Modern Foreign Languages, 4, $428-435$.

[7] Charles Horton Cooley, (1909). Social Organization: A Study of the Larger Mind, New York: Charles Scribner's Sons, 1-2.

[8] Lynch, A, (1996). Thought Contagion: How Belief Spreads Through Society, New York: Basic Books.

Yongmei Jiang was born in Qingdao, China in 1974. She received her M.A. degree in linguistics from The Ocean University of China in 2004.

She is currently a tutor in the School of Foreign Languages, Qingdao University of Science and Technology. Her research interests include psycholinguistics, translation and language teaching. 